(C) [2008] IEEE. Reprinted, with permission, from Guo, Youguang; Zhu, Jianguo; Zhong, Jin Jiang; Lu, Hai Yan; Jin, Jianxun. 2008, 'Measurement and Modeling of Rotational Core Losses of Soft Magnetic Materials Used in Electrical Machines - A Review', IEEE Transactions On Magnetics, Vol. 44, no. 2, pp. 279291. This material is posted here with permission of the IEEE. Such permission of the IEEE does not in any way imply IEEE endorsement of any of the University of Technology, Sydney's products or services. Internal or personal use of this material is permitted. However, permission to reprint/republish this material for advertising or promotional purposes or for creating new collective works for resale or redistribution must be obtained from the IEEE by writing to pubs-permissions@ieee.org. By choosing to view this document, you agree to all provisions of the copyright laws protecting it. 


\title{
Measurement and Modeling of Rotational Core Losses of Soft Magnetic Materials Used in Electrical Machines: A Review
}

\author{
Youguang Guo, Senior Member, IEEE, Jian Guo Zhu, Senior Member, IEEE, Jinjiang Zhong, \\ Haiyan Lu, Member, IEEE, and Jian Xun Jin
}

\begin{abstract}
In many situations, such as the cores of a rotating electrical machine and the $\mathrm{T}$-joints of a multi-phase transformer, the magnetic flux varies with time in terms of both magnitude and direction, i.e. the local flux density vector rotates with varying magnitude and varying speed. Therefore, the magnetic properties of the core materials under various rotational magnetizations should be properly investigated, modeled and applied in the design and analysis of electromagnetic devices with rotational flux. Based on the references selected from a huge amount of papers published by various researchers in the past century, this paper presents an extensive survey on the measurement and modeling of rotational core losses of soft magnetic materials used in electrical machines, particularly from the view of practical engineering application. The paper aims to provide a broad picture on the historical development of measuring techniques, measuring apparatus and practical models of rotational core losses.
\end{abstract}

Index Terms-Soft magnetic material, electrical machine, rotating flux, rotational magnetization, rotational core loss, rotational hysteresis loss, measuring technique, measuring apparatus, modeling, review.

\section{INTRODUCTION}

INCE THE invention of electrical machines in the $19^{\text {th }}$ N century, various magnetic materials have been employed as the cores of these electromagnetic devices. Typically, grain-oriented 3\% silicon-iron is used in transformer cores, and non-oriented silicon-iron is used for rotating electrical machine. The magnetic properties of the magnetic materials used have strong impact on the performance of these devices, and should be carefully characterized.

For evaluating and controlling the quality of magnetic materials, magnetic properties, such as $B$ (magnetic flux density) - $H$ (magnetic field strength) curves and core losses, are measured under one-dimensional (1-D) alternating and/or two-dimensional (2-D) rotating magnetic fluxes, according to

Manuscript received Sept. 5, 2007. This work was supported in part by the Australian Research Council under Large Grant No. A00104148.

Y. G. Guo, J. G. Zhu, and J. J. Zhong are with the Faculty of Engineering, University of Technology, Sydney, PO Box 123, Broadway, NSW 2007, Australia (e-mail: youguang@eng.uts.edu.au).

H. Y. Lu is with the Faculty of Information Technology, University of Technology, Sydney, PO Box 123, Broadway, NSW 2007, Australia.

J. X. Jin is with the Center of Applied Superconductivity, University of Electronic Science and Technology of China, Chengdu, Sichuan 610054, China. the application requirement. For example, in the core of a single-phase transformer, the magnitude of the local flux density vector varies with time sinusoidally along a direction. This type of flux is known as the alternating or 1-D flux. In other situations, such as the cores of a rotating electrical machine and the T-joints of a multi-phase transformer, the local magnetic flux density vector rotates within the electrical steel lamination plane. This type of flux is known as the 2-D rotating flux. Moreover, in some complex structure machines, e.g. claw pole and transverse flux machines, the magnetic field may rotate in the real three-dimension (3-D) space. It has been long known that the behavior of magnetic materials under a rotating flux is quite different from that under an alternating flux. Therefore, the magnetic properties of the core materials under different rotational magnetizations should be investigated, understood, properly modeled and employed in the design and performance analysis of electrical machines.

In a typical rotating electrical machine, both alternating and rotating flux density vectors exist. The measurement techniques under alternating flux have become standardized and the alternating magnetic property data of magnetic materials are normally provided by the manufactures. However, the magnetic properties under rotating flux are rarely available, and their measurement techniques and modeling approaches are still far from standardization.

In the past hundred years, a lot of work has been carried out by various researchers on the rotational core loss. Earlier effort focused on rotational hysteresis loss with purely circular rotational magnetic field. Since the 1960s, total core losses of magnetic materials under both circularly and elliptically rotating magnetic field excitations of different frequencies have been studied.

Based on a number of selected references, this paper aims to review the research work on the rotational core loss measurement and modeling conducted by various researchers, particularly from the view of engineering application. Section 2 briefs the understanding of core loss mechanism under rotating flux based on experimental measurements, and core loss modeling for practical appliance applications. In Section 3, four methods for measuring rotational core loss are described, and in Section 4, the techniques for measuring $B$ and $H$ are discussed. A development of measuring devices is presented in Section 5. Finally, the measuring system for 3-D vector magnetic property, developed by the authors, is briefed in Section 6. 


\section{UNDERSTANDING AND MODELING OF ROTATIONAL CORE LOSSES}

\section{A. Rotational Hysteresis Loss}

In 1896, Baily [1] conducted the first quantitative investigation on the rotational hysteresis loss of hard steel and soft iron. Fig. 1 shows the results obtained under rotating and alternating magnetic fields. For a range of flux density up to about $70 \%$ of the saturation, the rotational hysteresis losses in both hard steel and soft iron are larger than the alternating hysteresis losses. However, when the flux density further increases, the rotational hysteresis loss drops quickly and vanishes when the flux density reaches the saturation value, while the alternating hysteresis loss continues to increase. This indicates that the mechanism of the rotational hysteresis loss is different from that of its alternating counterpart.

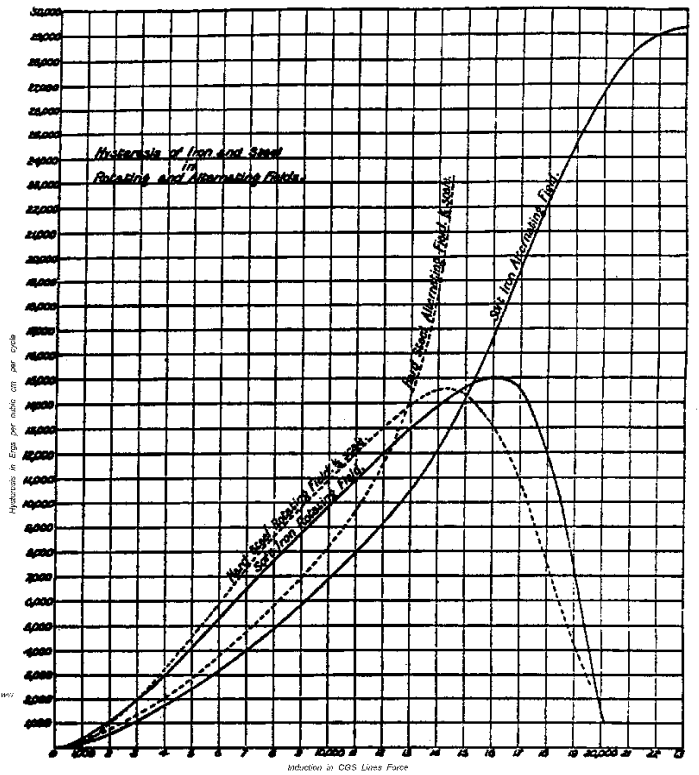

Fig. 1. Hysteresis losses of iron and steel obtained by Baily [1]

A qualitative explanation of the rotational core loss phenomenon was made based on the domain theory of ferromagnetism [2]. According to this theory, magnetization below the knee of the $B-H$ curve proceeds in steps, corresponding to the Barkhausen discontinuities, each step representing a reversal or sudden change in direction through $90^{\circ}$ of the spontaneous saturation in a domain. In addition, there may be a parallel reversible process, involving no hysteresis loss. For any particular domain, a sudden change in direction may be expected when a certain value of the field acting on it in the final direction of its magnetization has been reached. The total change in energy involved in this discontinuous change is then dissipated as hysteresis loss, a function of the volume of the domain, the saturation magnetization and the effective field strength.

At the knee of the $B-H$ curve, the field required to produce the discontinuous rotation has already been reached or exceeded for most of the domains, and these domains contribute further to the total magnetization on a further large increase of magnetic field strength $\boldsymbol{H}$, by a rotation of the spontaneous magnetization in the domains from the easy direction towards the direction of the applied field. There are some domains remaining, however, which are not acted upon until $\boldsymbol{H}$ has increased beyond its value at the knee. The contribution to the magnetization due to these domains is only that corresponding to their volume, but the contribution to loss is high on account of the high value of $\boldsymbol{H}$ at that point at which the sudden change in direction of the magnetization occurs. The loss will therefore rise steeply with the increase of magnetization.

Similar ideas may be applied to the portion of the loss curve near saturation. For a very high field, the spontaneous magnetization is always in the direction of the field, and as the field is rotated, it rotates smoothly and without the discontinuities which results in hysteresis loss. Hence, the hysteresis loss may then be zero.

In 1960, Archenhold et al. [3] measured the rotational hysteresis loss in grain oriented silicon iron for various orientations and thicknesses with rotating disk samples. The experimental results were discussed in terms of multi-domain concepts and of the Stoner-Wohlfarth single domain particle, of which the rotational hysteresis behavior had been evaluated.

The Stoner-Wohlfarth model is of a particle sufficiently small that it acts as a single domain with an angular dependence of the directional energy of the applied field caused either by strain, by crystal anisotropy, or by shape anisotropy. The variable part of the energy $E$ is

$$
E(\theta, \mathbf{H})=K \sin ^{2} \theta-\mu_{0} \mathbf{m}_{s} \bullet \mathbf{H}
$$

where $K$ is the anisotropy constant, $\theta$ the angle between the direction of easy axis and the magnetic moment $\boldsymbol{m}_{\boldsymbol{s}}$ of the particle, and $\boldsymbol{H}$ the magnetic field strength.

For rotational hysteresis, the $\boldsymbol{H}$ vector is taken as a constant and is rotated from an initial position aligned with the intensity vector, when there occurs a discrete energy jump for certain values of the field. This was derived and given by

$$
-\Delta W=\left(\frac{h^{2}+2}{3}\right)^{3 / 2}-\frac{7-h^{2}}{6}\left(\frac{4 h^{2}-1}{3}\right)^{1 / 2}
$$

where $h$ is the normalized crystalline anisotropy field, and the value must lie within the range of 0.5-1.0. However, there exists a significant discrepancy between the theoretical and experimental results. Similar investigations were performed by many other researchers and similar results were observed [48]. Phenomenological formulations are generally used to calculate the core losses of magnetic materials, which have no strong physical background but can provide a reasonably good accuracy for engineering application.

\section{B. Total Rotational Core loss}

In 1961, Kaplan [9] measured core losses of grain oriented (M-7) and non-grain oriented (M-19) silicon iron using cross samples under various flux conditions ranging from a pure alternating flux (only the magnitude of flux varies with time) to a pure rotating flux (only the direction of flux varies with time). It was found that the grain oriented iron was lower loss material under all flux conditions, but this is not always true. Since grain oriented steel sheet has stronger anisotropy due to 
texture which causes higher hysteresis loss, the rotational core loss in grain oriented steel sheets can be higher than that in non-oriented steel sheets.

In 1962, Strattant and Young [10] studied the power frequency losses in silicon iron alloy due to an elliptically rotating magnetic field with disk samples placed in the center of two perpendicular pairs of rectangular Helmholtz-type aircored coils, and predicted the losses approximately using a simple model without physical basis:

$$
\begin{aligned}
& P=P_{\text {hyst }}+P_{\text {eddy }} \\
& P_{\text {hsyt }}=P_{1}\left[\frac{B_{1}}{B_{s}}-\left(\frac{B_{1}}{B_{s}}\right)^{2}\right]+P_{2}\left[\frac{B_{2}}{B_{s}}-\left(\frac{B_{2}}{B_{s}}\right)^{2}\right] \\
& P_{\text {eddy }}=P_{3}\left[\left(\frac{B_{1}}{B_{s}}\right)^{2}+\left(\frac{B_{2}}{B_{s}}\right)^{2}\right]
\end{aligned}
$$

where $B_{1}$ and $B_{2}$ are the major and minor axis flux densities, $B_{s}$ is the saturation flux density, $P_{1}$ and $P_{2}$ are the alternating hysteresis losses in the major and minor axes for peak flux densities of $B_{s}$, respectively, and $P_{3}$ is the eddy current loss for a peak flux density of $B_{s}$. However this model only simulates the core loss with elliptically rotating magnetic fields, while the basic physical phenomena were not described.

In 1965, Boon and Thompson [4] measured alternating and rotational core losses at $50 \mathrm{~Hz}$ under various flux densities for both hot rolled and cold rolled 3\% silicon iron in a square sample of $0.33 \mathrm{~mm}$ laminations, using an improved thermometric method, and found that the ratio of rotational loss to alternating loss at $50 \mathrm{~Hz}$ in four-square silicon iron was about 2:1 over a wide range of flux densities (except at a high flux density), as illustrated in Fig. 2.

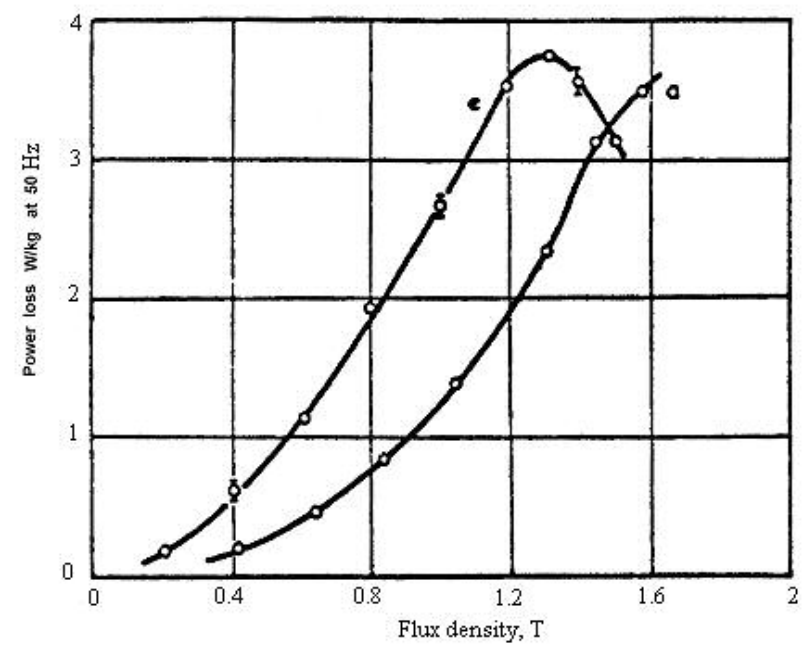

Fig. 2. Core losses in $0.33 \mathrm{~mm}$ hot-rolled $3 \%$ silicon iron sheet at $50 \mathrm{~Hz}$ : 'a' alternating, and 'e' - rotational [4].

Moses and Thomas [11] studied the localized rotational power loss in the T-joints of three-phase transformer in 1973. Also, a large amount of research on rotational core loss has been conducted by many other researchers, as reported in [1225]. For example, Enokizono et al. [21-23] measured rotational core losses in non-oriented and grain oriented steel sheets using a square sheet tester.

In 1990, Fiorillo and Rietto [24-25] reported the experimental results of rotational core loss in $3.2 \%$ nonoriented $\mathrm{SiFe}$ at different magnetization frequencies up to 50 $\mathrm{Hz}$, as depicted in Fig. 3, measured by their tester using fixed disk samples. By plotting the losses against frequency as illustrated in Fig. 4, it was shown that the rotational core loss $P_{r}$ could also be separated into rotational hysteresis $P_{h r}$, classical eddy current $P_{e r}$, and anomalous losses $P_{a r}$, similar to the case of alternating core loss. This formula has no physical basis and the domain wall behavior is ignored.

$P_{r}=P_{h r}+P_{e r}+P_{a r}$

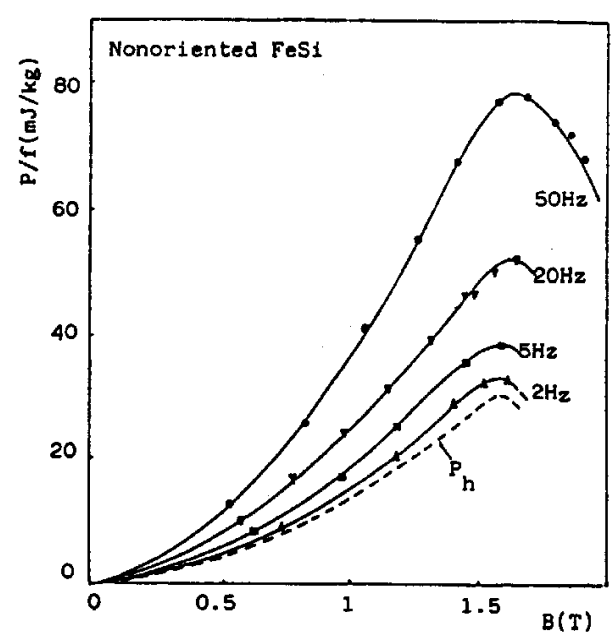

Fig. 3. Specific rotational core loss per cycle vs. peak flux density in a $3.2 \%$ SiFe non-oriented sheet, $0.637 \mathrm{~mm}$ thick, at different frequencies, where $P_{h}$ is the rotational hysteresis loss obtained by extrapolating the specific rotational core loss vs. frequency curves to zero frequency [24].

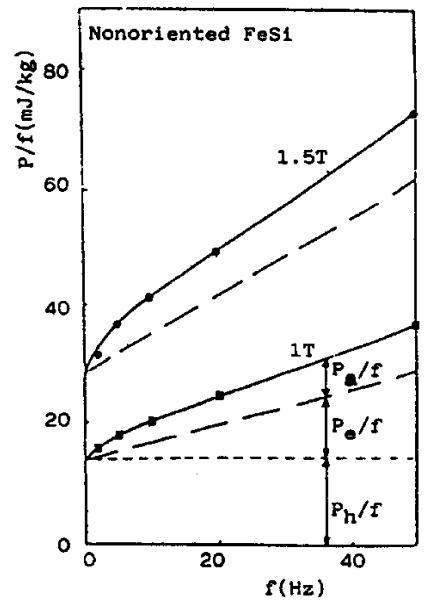

Fig. 4. Specific rotational core loss vs. frequency in a $3.2 \%$ non-oriented sheet at $1 \mathrm{~T}$ and $1.5 \mathrm{~T}$, where $P_{h}$ is the rotational hysteresis loss, $P_{e}$ the eddy current loss, and $P_{a}$ the anomalous loss [24].

A phenomenological approach to the rotational anomalous loss $P_{a}$ was proposed in [25] as following

$P_{a r}=C_{a r}(f B)^{1.5}$

where $C_{a r}$ is the anomalous loss coefficient, $f$ the magnetization frequency, and $B$ the magnitude of flux density. 
This formula is essentially the same as the one for alternating anomalous loss, except that the rotational anomalous loss coefficient $C_{a r}$ is a function of flux density $B$, while the one for alternating anomalous loss is a constant, and $C_{a r}$ would eventually drop to zero when the material was saturated and all domains disappeared.

From 1998, the authors [26-30] of this paper systematically measured the core losses of soft magnetic composite (SMC) materials, which are specially developed for application of electrical machines, particularly those with complex structures and three-dimensional (3-D) flux paths [31]. For both alternating and rotational core loss modeling, the empirical three-term formula (6) is used to separate the loss into three components: hysteresis, eddy current and anomalous losses.

Based on a large amount of experimental results on the SMC sample under alternating or circularly rotating flux excitations with various flux density magnitudes and frequencies, the core loss coefficients can be deduced using the curve-fitting technique.

In a rotating machine, the flux density locus in each element can be considered as elliptically rotating with harmonics (including purely circular and purely alternating) [32]. A series of magnetic field FEAs are conducted to find out the major and minor axis flux densities $B_{k m a j}$ and $B_{k m i n}$, then the total core loss can be predicted by

$P_{t}=\sum_{e=1}^{N_{e}} \sum_{k=1}^{\infty}\left[P_{r k} R_{B k}+\left(1-R_{B k}\right)^{2} P_{a k}\right]$

where $N_{e}$ is the number of elements of core material, $R_{B k}=B_{k m i n} / B_{k m a j}$ the axis ratio of the $k$-th harmonic ellipse, $P_{a k}$ the alternating loss with flux density $B_{k m a j}$, and $P_{r k}$ the purely rotational loss.

\section{Methods For Measuring Rotational Core Loss}

In the past hundred years or so, considerable progress has been achieved in terms of measuring techniques and systems. There are four major methods for measuring the rotational core loss:

\section{A. Torque-metric Method}

The torque-metric method is usually used in apparatus which use disk or ring sample, as will be described later. The torque due to rotational core loss occurring in the sample is measured by using mechanical torque meters [1-3], or calculated from the variation of sample angular speed [14].

The advantages of this method are the direct reading of the torque corresponding to rotational core loss from the torque meter, and the ability to measure rotational core loss with high flux density. The disadvantage is the difficulty of torque meter construction owing to the complicated mechanics.

\section{B. Thermometric Method}

In the thermometric method, the temperature of the sample is obtained by thermocouples, thermistors, or thermoviewers. The rotational core loss is proportional to the initial rate of the sample temperature rise if no cooling process is involved, namely,
$P_{r}=C \frac{d \theta}{d t}$

where $P_{r}$ is the specific rotational core loss in $\mathrm{W} / \mathrm{kg}, C$ the specific heat of the sample material, $\theta$ the temperature of the sample, and $t$ the time instant.

This is a very versatile method that has been widely used in apparatus using various types of sample, such as square [4], disc [24], ring [33], and cross [34], with various types of rotating magnetic field. It is also able to measure localized core loss at the $\mathrm{T}$ joints of a three-phase transformer core [11].

The major shortcomings of this method are the difficulties of installation and calibration of thermosensors, and isolation against the surrounding. It is therefore being more and more replaced by the field-metric method.

\section{Field-metric Method}

In the field-metric method, rotational core loss is calculated from the measured magnetic field strength $\boldsymbol{H}$ at the sample surface and flux density $\boldsymbol{B}$ inside the sample. This method features high accuracy and great versatility. Moreover, the set of measured instantaneous $\boldsymbol{H}$ and $\boldsymbol{B}$ values can yield more desirable information, such as various loss contributions, the loci of $\boldsymbol{H}$ and $\boldsymbol{B}$ vectors, and harmonics etc. The main disadvantages are the difficulties of manufacture, calibration, and installation of $\boldsymbol{B}$ and $\boldsymbol{H}$ sensors, and the sensitivity to preamplifier phase angle errors.

For the evaluation of rotational core loss, there exist two formulas, which are here referred as the field-metric method type I and type II, respectively. Type I calculates the total specific core loss $P_{t}$ by using the Poynting theorem as shown in $[9,23,35-38]$ :

$P_{t}=\frac{1}{T \rho_{m}} \int_{0}^{T}\left(\mathbf{H} \bullet \frac{d \mathbf{B}}{d t}\right) d t=\frac{1}{T \rho_{m}} \int_{0}^{T}\left(H_{x} \frac{d B_{x}}{d t}+H_{y} \frac{d B_{y}}{d t}\right) d t$

where $T$ is the time period of magnetization, $\rho_{m}$ the mass density of sample, $H_{x}, H_{y}, B_{x}, B_{y}$ are the $X$ and $Y$ components of $\boldsymbol{H}$ and $\boldsymbol{B}$, respectively.

In the field-metric method type II [15], the torque per unit volume due to the rotational core loss in the sample is calculated by

$T_{r}=\mu_{0}|\mathbf{H} \times \mathbf{M}|=\mu_{0} H M \sin \alpha=\mu_{0} H M_{\perp}$

where $\mu_{0}$ is the magnetic permeability of vacuum, $\boldsymbol{M}$ the magnetization, $\alpha$ the angle between $\boldsymbol{H}$ and $\boldsymbol{M}$ vectors, and $M_{\perp}$ the component of $\boldsymbol{M}$ perpendicular to $\boldsymbol{H}$.

\section{Watt-metric Method}

The Watt-metric method differs from the field-metric method in that $\boldsymbol{H}$ is determined by the magnetization current [36], and is widely used in the Epstein frames or single sheet testers for alternating core loss measurement [39]. Initially, $\boldsymbol{H}$, $\boldsymbol{B}$ and core loss were measured by ammeters, voltmeters, and wattmeters, respectively. That is why this method is known as the watt-metric method. With the development of digital techniques, $\boldsymbol{H}$ and $\boldsymbol{B}$ waves can be readily obtained in numerical form, and core losses can then be calculated by 
using (10). An outstanding advantage of this method is the simplicity in determining $\boldsymbol{H}$.

In apparatuses for rotational core loss measurement, this method can only be applied to the vertical yoke single sheet tester [40], because of the absence of air gaps between the sample and the yokes. Compared with alternating core loss testers, the magnetic flux paths in the sample and the yoke system of the rotational core loss testers are not well defined. This causes excessive systematic error in magnetic field strength measurement by applying Ampere's law. Since the flux density is not uniformly distributed in the sample, the magnetic flux density is measured in the center of the sample by coils wound through small holes, or $\boldsymbol{B}$ tips. The accuracy of measurement strongly depends on the structure of the yoke and the shape of the sample, which define the path of the magnetic flux.

\section{TECHNIQUES FOR MEASURING $H$ AND $B$}

\section{A. Magnetization Current Method}

When the magnetic circuit of a core loss tester satisfies: (a) the magnetic flux path inside the sample is well defined, (b) there are no dissipative processes outside the sample, and (c) there is no magnetic potential drop outside the sample, the magnetic field strength $H$ can be determined from the magnetization current $i$ by applying Ampere's law:

$$
H=N i / l_{m}
$$

where $N$ is the number of turns of the excitation winding, and $l_{m}$ the mean length of magnetic flux path.

This method is widely used in apparatus for alternating core loss measurement, such as annular rings, Epstein frames, and single sheet testers [39]. If used in rotational core loss testers, however, this method is not accurate, since the magnetic flux paths in the specimen are vague [40].

\section{B. Sensing Coil Method}

\section{1) Conventional $\mathrm{H}$ coil}

The tangential component of magnetic field strength at specimen surface can be measured by a thin search coil placed on the surface, as illustrated in Fig. 5.
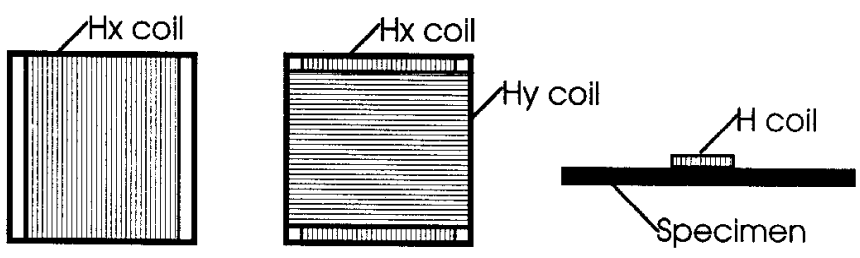

Fig. 5. (a) 1-D $\boldsymbol{H}$ coil, (b) 2-D $\boldsymbol{H}$ coil, and (c) position of $\boldsymbol{H}$ coil.

When the magnetic field is parallel to the surface, the magnetic field strength can be calculated by

$$
H=\frac{1}{\mu_{0} K_{H}} \int V_{H} d t
$$

where $K_{H}$ is the coil coefficient determined by calibration, and $V_{H}$ the terminal voltage of the $H$ sensing coil.
This method is commonly used in both alternating and rotational core loss testers, and can yield accurate results if the magnetic field on the specimen surface is uniform. In square specimen rotational core loss testers with horizontal yokes, however, the magnetic field varies significantly with the distance between the specimen surface and the $\boldsymbol{H}$ coils, as observed by several researchers [37-38, 41-42]. In order to reduce the error, the sensing coils must be made extremely thin and installed as close to the specimen surface as possible, but this is often very difficult.

\section{2) Two H coil arrangement}

To reduce the error caused by the variation of magnetic field with the distance above the specimen surface, a two $\boldsymbol{H}$ coil arrangement, as shown in Fig. 6, can be used and the field strength at the specimen surface can be extrapolated by

$H=\frac{d_{2} H_{1}-d_{1} H_{2}}{d_{2}-d_{1}}$

where $H_{1}$ and $H_{2}$ are the magnetic field strength measured by $H$ coil $\# 1$ and \#2, $d_{l}$ and $d_{2}$ the distances of two $\boldsymbol{H}$ coils away from the specimen surface.

Both numerical analysis [41] and experimental measurement $[37,43]$ have shown that the linear extrapolation is a reasonable approximation, when two $\boldsymbol{H}$ coils are placed not far away from the specimen.

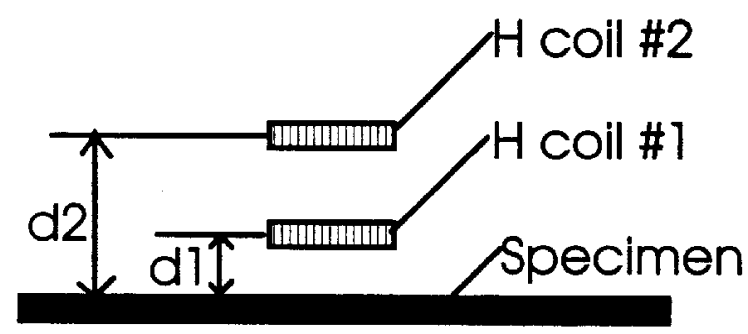

Fig. 6. Two $\boldsymbol{H}$ coil arrangement

3) The Rogowski-Chattock coil

Another choice of higher accuracy than the conventional $\mathbf{H}$ coil is the Rogowski-Chattock coil (also known as magnetic potentiometer) [44-46], as shown in Fig. 7.

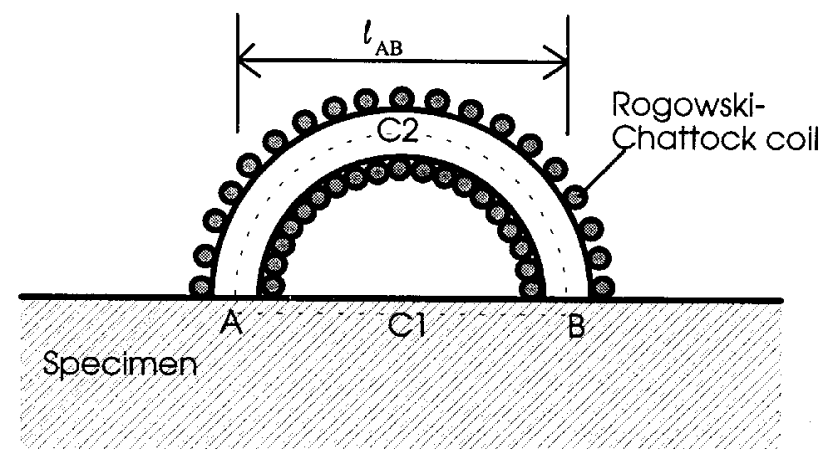

Fig. 7. The Rogowski-Chattock coil

The principle of the Rogowski-Chattock coil is based on the existence of the scalar magnetic potential $V_{m}$ in the absence of currents. The magnetic potential difference between two points 
$A$ and $B$ can be determined by

$$
V_{m A}-V_{m B}=\int_{C 1} \mathbf{H} \bullet d l=\int_{C 2} \mathbf{H} \bullet d l
$$

If the magnetic field is uniform between point $A$ and $B$, the line integral of $\boldsymbol{H}$ along path $C 1$ can be calculated as

$$
\int_{C 1} \mathbf{H} \bullet d l=H l_{A B}
$$

where $l_{A B}$ is the distance between point $A$ and $B$.

When the magnetic field varies with time, the induced terminal voltage of the coil is

$$
V_{H}=\frac{d \lambda}{d t}=\mu_{0} A_{H} n \frac{d}{d t} \int_{C 2} H \bullet d l
$$

where $\lambda$ is the total flux linkage, $A_{H}$ the cross sectional area, and $n$ the number of turns per unit length of the coil. Substituting (15) and (16) into (17) yields

$V_{H}=\mu_{0} A_{H} n \frac{d\left(H l_{A B}\right)}{d t}=\mu_{0} K_{H} \frac{d H}{d t}$

where $K_{H}=A_{H} n l_{A B}$ is the coil coefficient, which can be determined by calibration. Therefore, the magnetic field strength $\boldsymbol{H}$ on the specimen surface can also be obtained by the time integral of $V_{H}$.

Because both ends of the coil can be installed very close to the specimen surface, correct $H$ can be detected, and higher sensitivity can be achieved by a larger coil coefficient $K_{H}$ which is proportional to the number of turns per unit length of the coil.

\section{4) B coil}

Magnetic flux density $\mathbf{B}$ vector in core loss testers can also be measured by using a sensing coil [37]. When the magnetic flux density is uniformly distributed over the cross section of a sample, the sensing coil can be wound around the whole sample, as illustrated in Fig. 8(a). If the flux density is nonuniform over the cross section of a sample, the sensing coil can then be threaded through small holes at the position of interest, as shown in Fig. 8(b).

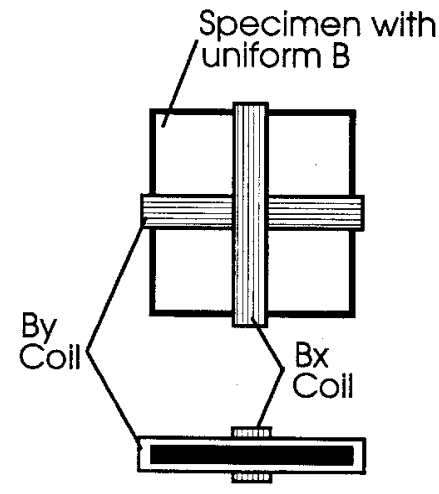

(a)

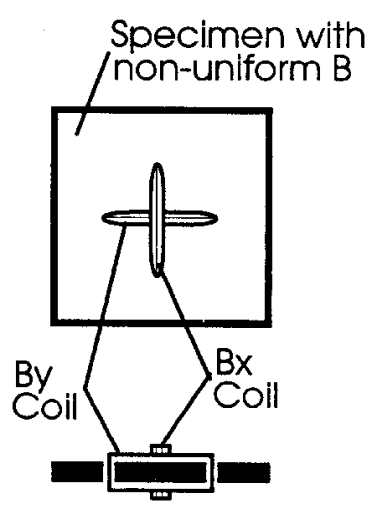

(b)
Fig. 8. $\boldsymbol{B}$ coil settings for specimens with (a) uniform $\boldsymbol{B}$, and (b) non-uniform $\boldsymbol{B}$.

\section{Hall Elements}

This method makes use of the Hall effect, which occurs in any conductor carrying a current in the presence of a transverse magnetic field. In semiconductors, this effect is much larger than in metals. If there is a current $i$ in a plateshaped semiconductor, as illustrated in Fig. 9, two opposite points $a$ and $b$ will be the same potential in the absence of a magnetic field. When a magnetic field $\boldsymbol{H}$ exists at right angle to the plate, the current path is distorted, and an electromagnetic force $(e m f) e_{H}$ is developed between $a$ and $b$. The magnitude of the magnetic field strength can be determined by

$H=\frac{e_{H} t}{R_{H} i}$

where $t$ is the thickness of the plate, and $R_{H}$ the Hall constant, which is a property of the material.

Because of the difficulty of installation, this method is not commonly used in rotational core loss testers, except the one using a rotating disk sample developed by Flanders in 1985 [16].

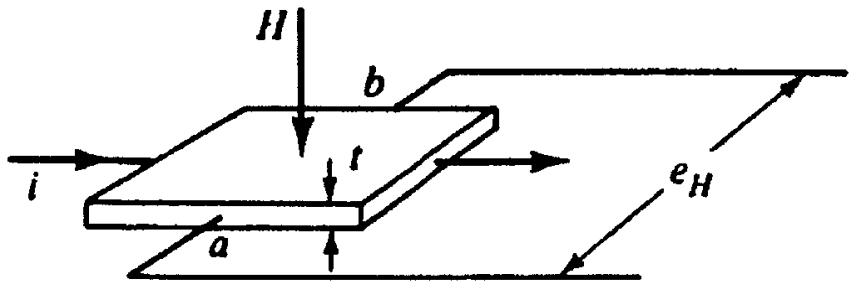

Fig. 9. Relationship between magnetic field, current, and emf in the Hall effect.

\section{B Tips}

This method was first developed by Werner in 1949 [47], and used for rotational core loss measurement by Kaplan in 1961 [9], Brix in 1982 [35], and Sievert in 1990 [48]. As illustrated in Fig. 10, the induced electromotive force $V$ is measured between two needle tips placed a certain distance apart in contact with the specimen surface.

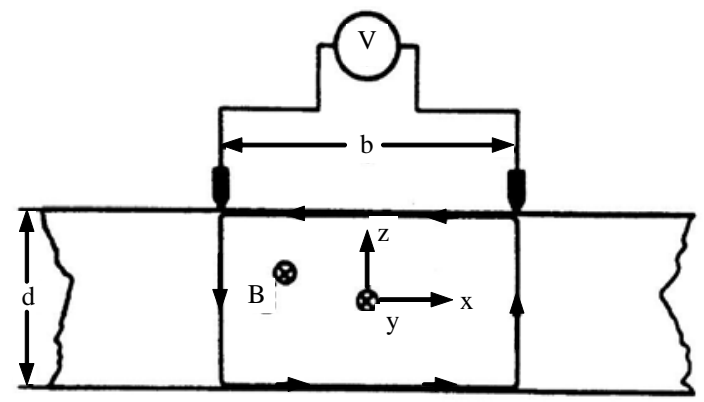

Fig. 10. Principle of measuring one component of $\mathbf{B}$ using tips.

If the specimen is a thin lamination, by Maxwell's equation, the measured voltage can be approximately calculated by

$V=\frac{b d}{2} \frac{d}{d t} B_{y}$

where $d$ is the thickness of the sample, $b$ the distance between 
two potential tips, and $B_{y}$ the $Y$ component of magnetic flux density in the specimen. Therefore

$$
B_{y}=\frac{2}{b d} \int V d t
$$

Compared with the $\mathbf{B}$ sensing coils, $\mathbf{B}$ tips are more suitable for batch measurements, but limited to conducting materials. Essentially, this method is equivalent to a one-turn search coil. In practical measurement, high quality preamplifiers are required, since the voltage signal obtained from the tip is very weak. It is also very difficult to exclude stray fluxes through the air, which may become significant when the specimen size is small. Therefore, the sensitivity of this method is lower than that of the $\mathbf{B}$ search coil.

\section{DeVElopment of MeAsuring Apparatus}

\section{A. Disk and Ring Samples}

In earlier measurements, disk samples were commonly used. A typical set up was developed by Brailsford in 1938, as shown in Fig. 11 [2]. The rotational hysteresis loss was determined by measuring the torque due to rotational hysteresis, and hence known as torque magnetometer. To eliminate the torque caused by anisotropic effect, a stack of several disks with the easy directions uniformly oriented were adopted, and the sample was rotated both clockwise and anticlockwise. The average torque curve of these two rotating directions would be the torque curve only due to the rotational hysteresis loss. For accurate torque measurement, the friction of the mechanical system should be kept as small as possible.

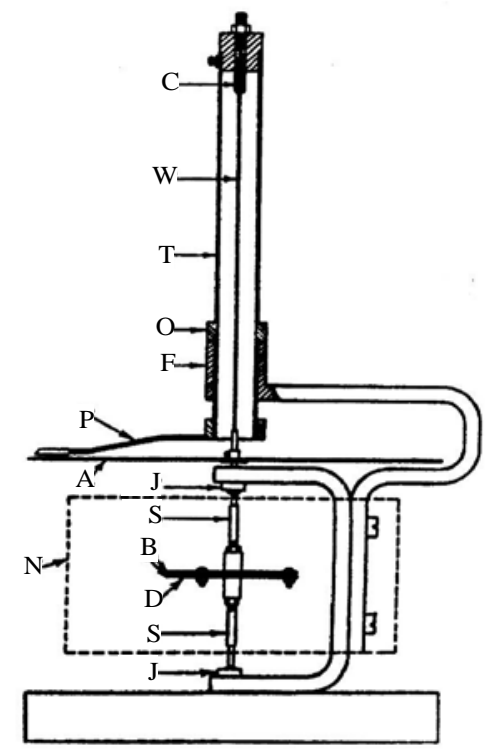

Fig. 11. Torque magnetometer built by Brailsford [2]

In 1967, Flanders developed a rotating sample magnetometer [49], which could be used for multiple purposes, such as the measurements of magnetic moment, rotational hysteresis, spin flop, and properties related to anisotropic energy, i.e. anisotropic constants, anisotropic susceptibility, and anisotropic spontaneous magnetization. The measurements were performed on a single-piece rotating sample. Sensing coils were used to detect the magnetization perpendicular to the applied magnetic field. The field metric method type II was used to evaluate the torque due to rotational hysteresis.

To minimize the pickup error due to variations in magnetic field or to coil motion relative to magnetic field strength $\boldsymbol{H}$, a set up of two coils connected in series opposition was employed. This method gives better results than the torque magnetometer made by Brailsford, since the effect of mechanical friction has been removed. This magnetometer was further improved by replacing the sensing coils with Hall elements in 1985 [16].

Besides the rotating disk samples, a fixed disk sample was used by Fiorillo and Reitto in 1988 and 1991 [24, 50]. Fig. 12 shows the layout of the equipment and the cross sectional view of a sample. It consists of a number of disks of scaled diameters, cut from the same lamination, and superposed to emulate an oblate ellipsoid. The sample was placed in a cylindrical plexiglass chamber, which could be evacuated as required by the thermometric method. The rotating field was generated by a three phase motor stator. Both the thermometric and field-metric type I methods were used for the determination of rotational core loss.

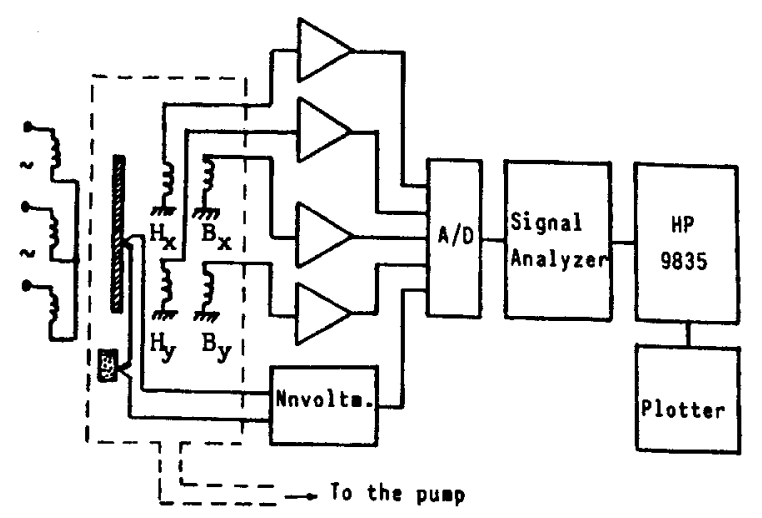

(a)

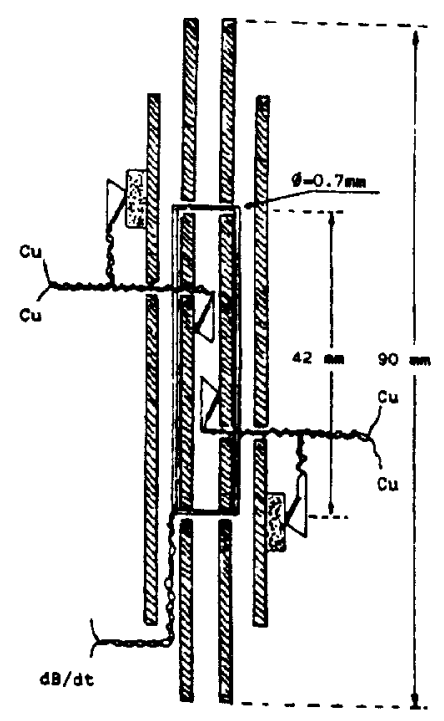

(b)

Fig. 12. (a) Equipment layout, and (b) cross sectional view of a sample [50] 
In 1987, Reisinger developed an apparatus using a stack of ring sample as shown in Fig. 13 [33]. The torque-metric and thermometric methods were adopted. Essentially, this apparatus has the same features as those using disk samples described above. However, the sample preparation and the flux control are not convenient.

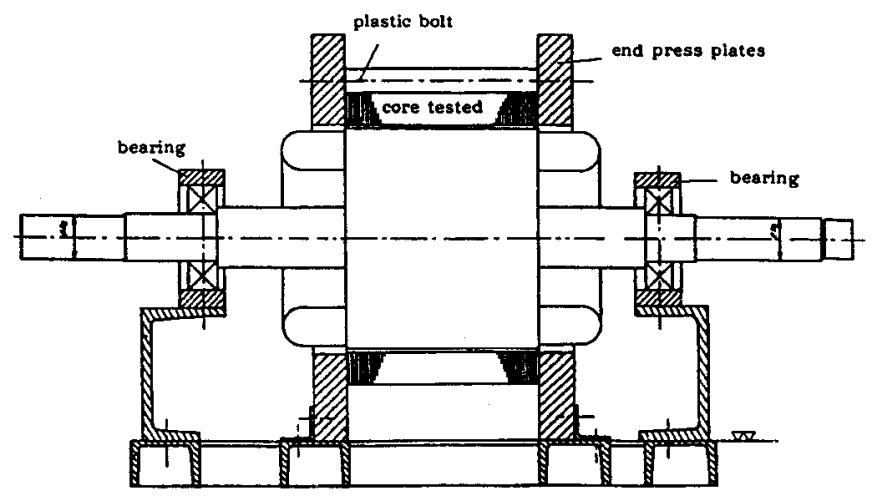

(a)

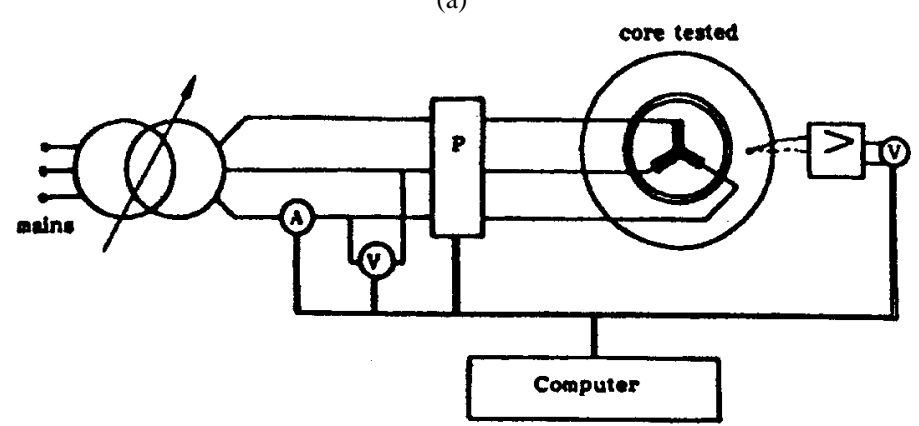

(b)

Fig. 13. Rotational core loss measuring system using a stack of ring samples built by Reisinger, (a) measuring apparatus, and (b) circular diagram [33]

The major disadvantages of these disk sample methods are: (a) Since the magnetic flux density is not controlled by feedback, the flux density fluctuates according to the anisotropic permeability of the sample. This is particularly serious when grain oriented materials are under test. (b) The magnetic field is not uniform within the sample. This will affect the precision of the measurement. (c) Disk samples cannot be conveniently used for testing under various flux conditions, such as elliptically rotating magnetic fields. In practice, it is often required to study the core loss under a rotating field of variable magnitude.

\section{B. Cross and Strip Samples}

On the other hand, the cross samples do not have these problems. In 1973 Moses and Thomas measured the rotating magnetic flux and the rotational core loss in silicon iron laminations with cross samples as shown in Fig. 14 [34]. The 2-D magnetic field was generated by the excitation windings wound on the cross sample. The rotational core loss was measured by the sensing coils wound through very small holes in the centre of the cross, while the magnetic field strength was determined from the magnetization current. In this set-up, there was no flux density feedback control. Therefore, in grain oriented samples, the magnitude of flux density was not kept constant.

In 1978, Basak and Moses [13] studied the sensitivity to mechanical stress of rotational power loss in silicon iron with cross samples and flux density feedback. Fig. 15 illustrates their system.

In 1982, Brix, Hemple, and Schroeder [35] built a fully computerized control and measurement system with cross samples as shown in Fig. 16. In this system both magnetic field strength $\boldsymbol{H}$ and flux density $\boldsymbol{B}$ were obtained by the sensing coils, and the power loss was determined by the field-metric method type I.

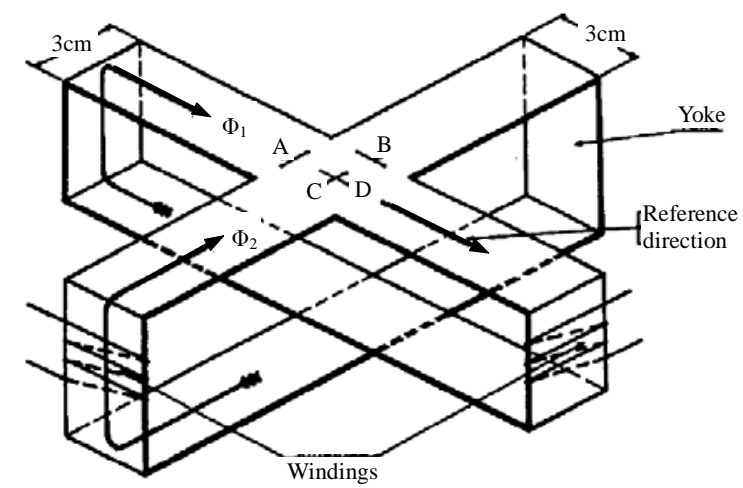

Fig. 14. Cross sample used by Moses and Thomas [34]

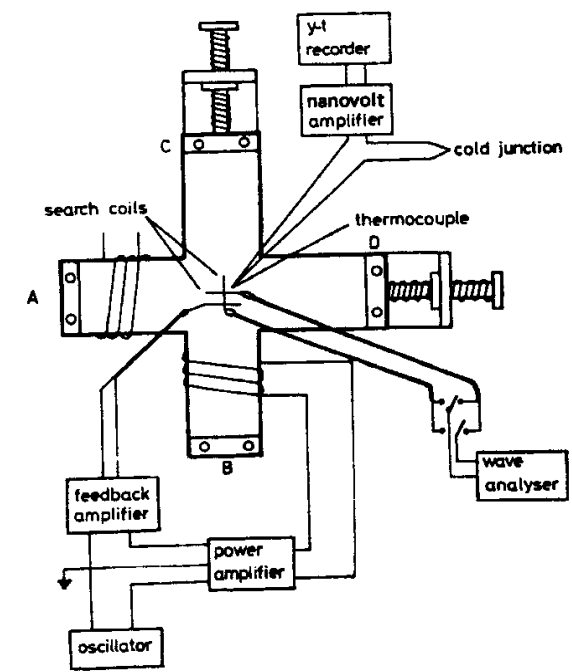

Fig. 15. Apparatus for measuring rotational core loss under complex stress, where A and B are fixed ends, C and D spring loaded ends [13]

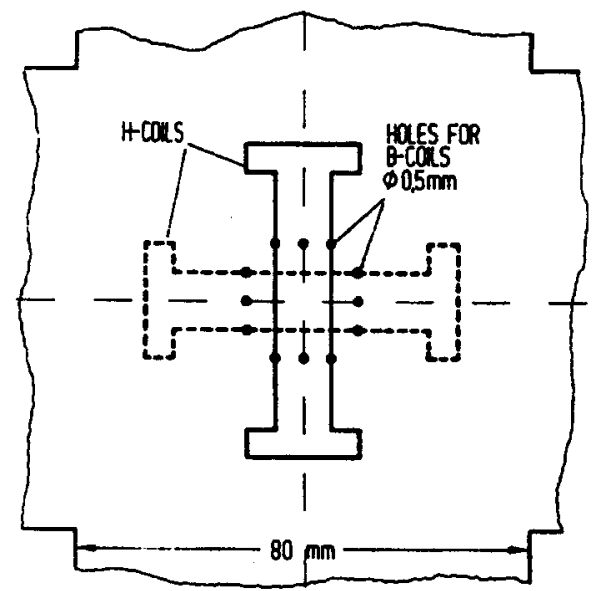

Fig. 16. Block diagram of a rotational core loss measuring system using cross samples [35] 
In 1985, Sasaki, Imamura, Takada, and Suzuki built a measuring system using a single Epstein strip [51]. Sensing coils were used to measure magnetic field strength and flux density, and the core loss was calculated by the field-metric method type I. This system is quite similar to those using cross samples. The configuration and the block diagram are illustrated in Fig. 17.

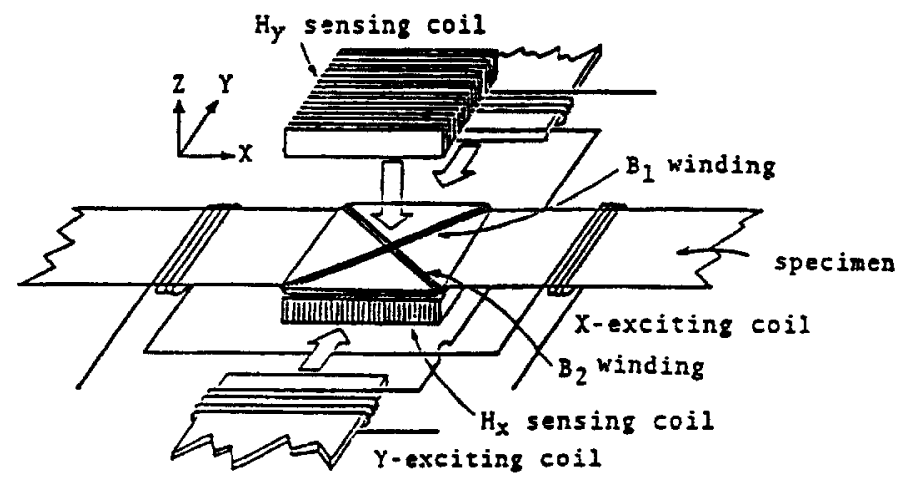

(a)

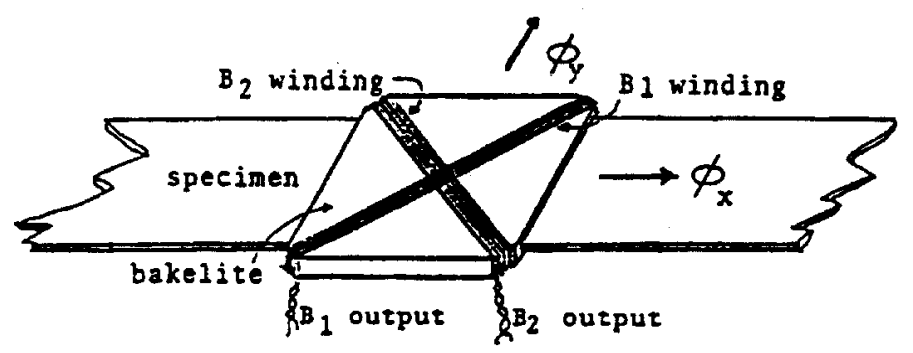

(b)

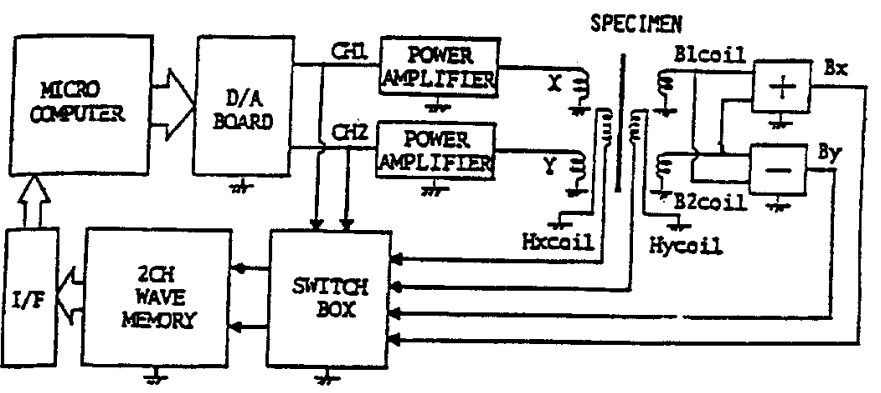

(c)

Fig. 17. Rotational core loss tester using an Epstein strip: (a) configuration, (b) magnetic flux search coils, and (c) system diagram [51].

\section{Square Samples}

In 1984, Brix, Hempel, and Schulte [52] found that the magnetic field was more uniform in a square sample than in a cross sample, and developed a tester using square samples. This kind of tester system is briefly called square sample tester. In this system, $\boldsymbol{B}$ tips were exploited for detecting magnetic flux density. Fig. 18 illustrates the whole arrangement and the sensor.

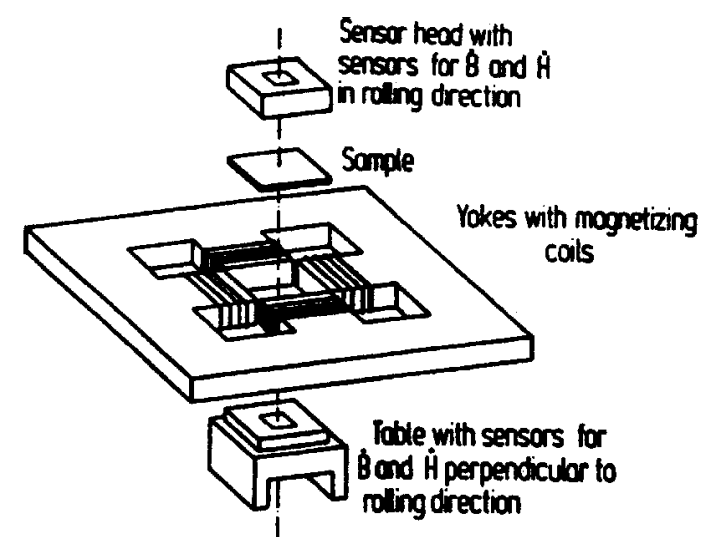

(a)

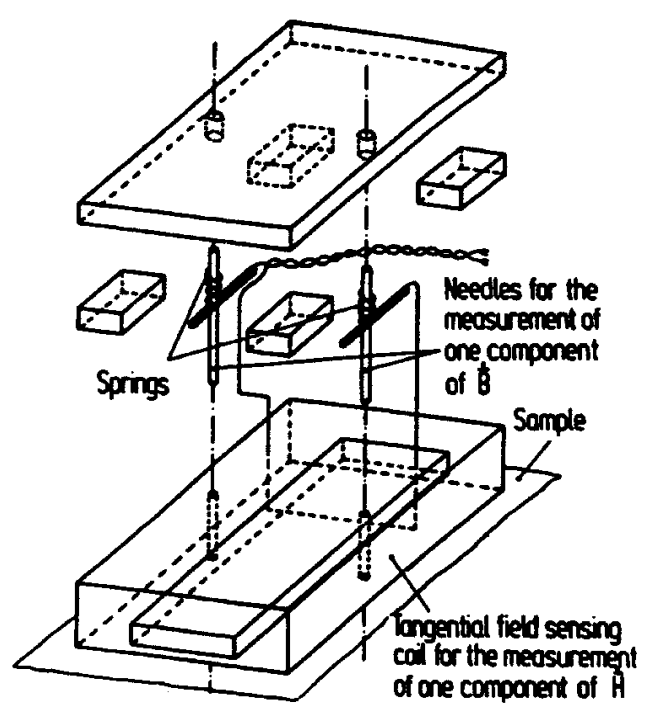

(b)

Fig. 18. Rotational core loss tester using square samples: (a) arrangement of yoke, sample and sensors, and (b) details of sensor head for one component of $\boldsymbol{H}$ and $\boldsymbol{B}$ [52].

In 1989, Enokizono and J. Sievert developed a very flexible system consisting of a horizontal magnetic circuit with a square single sheet sample and adjustable air gaps, an analog electronic circuit for flux density feedback control, and a computer which performed function generation and data acquisition in rotational core loss measurement [21, 41-42, 48, 53-54]. Fig. 19 shows the configuration and the circuit diagram. The magnetic field strength was picked up by conventional surface $\boldsymbol{H}$ sensing coils. For flux density measurement, $\boldsymbol{B}$ sensing coils threaded through small holes in the centre of square sample were adopted by Enokizono [53], while $\boldsymbol{B}$ tips were used by Sievert [54], which is more convenient for batch measurements.

This system can be used to examine the behavior of ferromagnetic materials under either rotational or alternating magnetic field. Measurements on rotational core losses of various electrical steels and dynamic magnetostriction under rotational field have been performed with this system. 


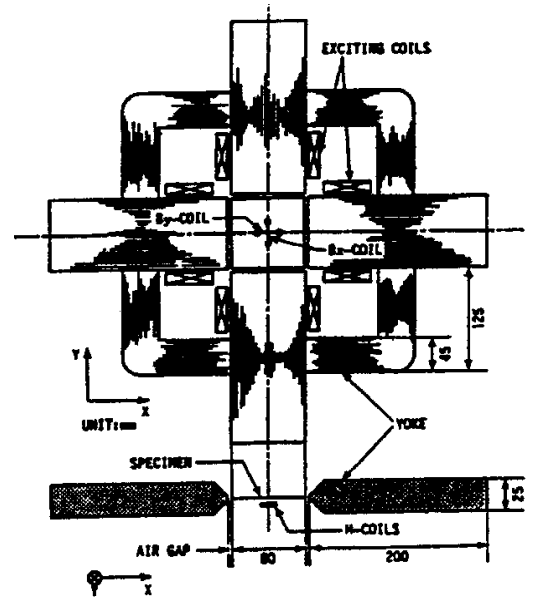

(a)

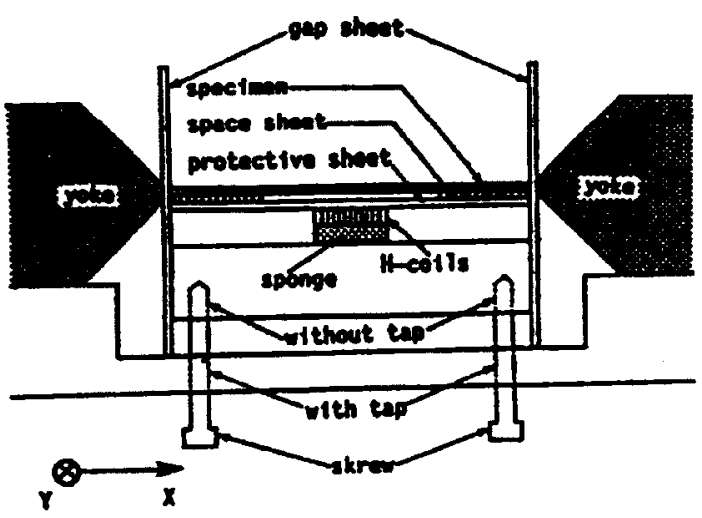

(b)

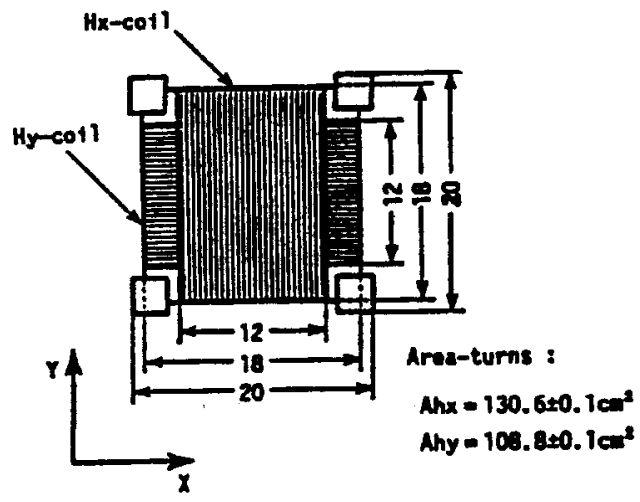

(c)

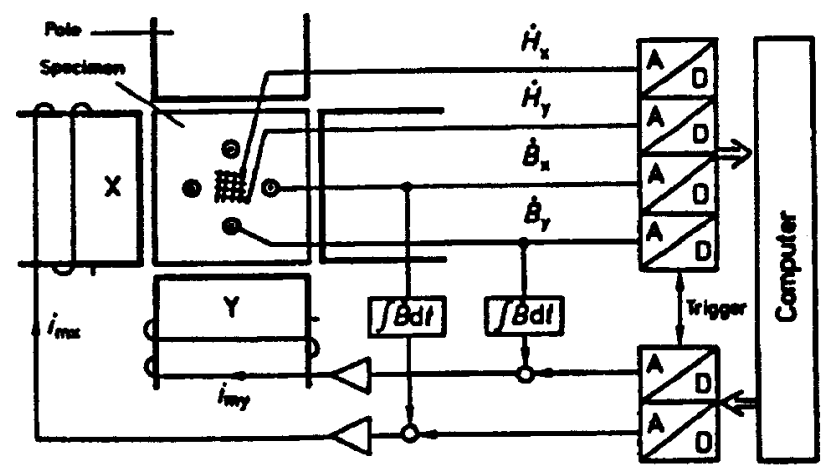

(d)

Fig. 19. Rotational core loss measuring system built by Enokizono and Sievert: (a) magnetic circuit and $\boldsymbol{B}$ sensing coils, (b) setting of $\boldsymbol{H}$ sensing coils, (c) $\boldsymbol{H}$ sensing coils, and (d) system diagram and $\boldsymbol{B}$ tips [42].
In 1992, Zhu developed a system comprising of a horizontal magnetic circuit with a square single sheet sample, feedback control system, and the digital signal processing for the specification of flux density waveforms and the data acquisition at University of Technology, Sydney (UTS) [37]. Fig. 20 illustrates schematically the square sample tester and the whole testing system. By using this tester, the authors of this paper have systematically investigated the alternating and rotational magnetic properties of several magnetic materials, including measurement, modeling and application in analysis of rotating motors [8, 26, 29, 37, 43, 46, 55].

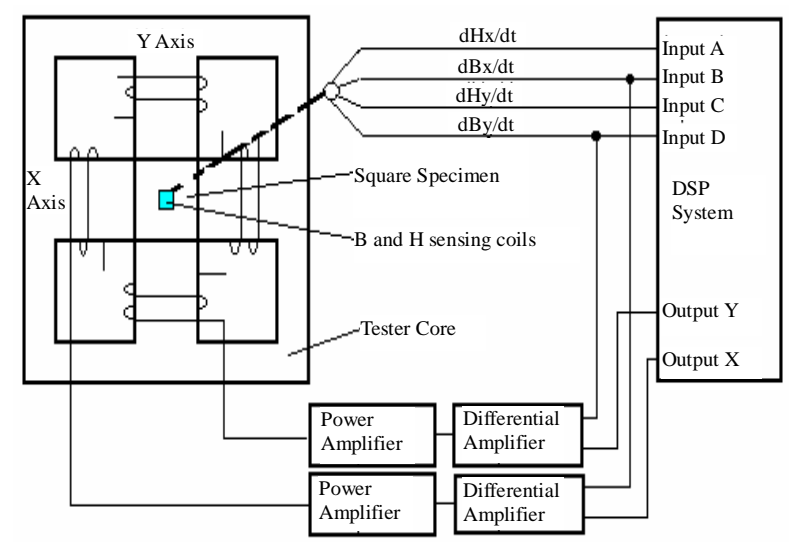

(a)

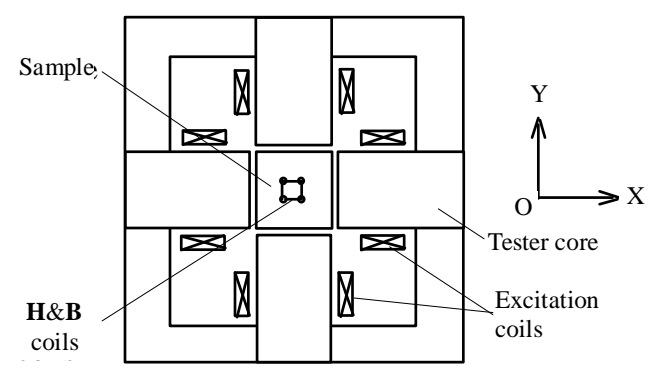

(b)

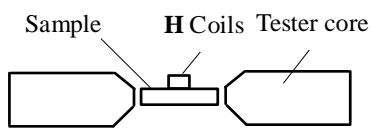

(c)

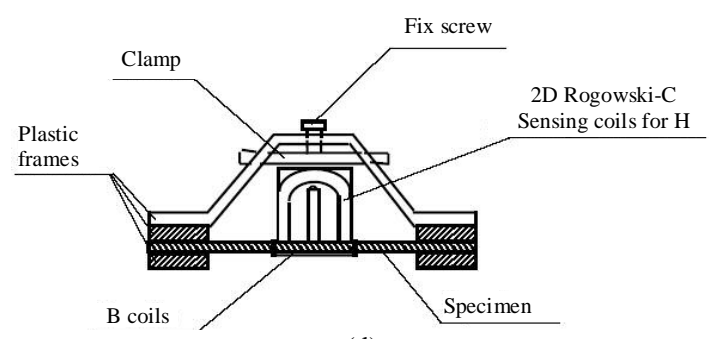

(d)

Fig. 20. Schematic illustrations: (a) the block diagram of the rotational core loss testing system at UTS, (b) the square specimen tester, (c) position of the sample between the magnetization poles, and (d) position of sample and Rogowski-Chattock sensing coils [37].

In conclusion, the computer controlled square sample testing system is more advantageous than other types in the following aspects:

(1) Since the magnetic fluxes in the two perpendicular directions are controlled by feedback, the generated 
magnetic field excitations of various complex magnetic flux patterns can be used to simulate the actual situation happening in electrical machines where the magnetic field is rotating with either constant or varying magnitude.

(2) The measurement is carried out in the center of the specimen where the field appears to be most uniform, which leads to more accurate results.

(3) More information can be obtained from the measured $\boldsymbol{B}$ and $\boldsymbol{H}$ waveforms which helps in understanding the mechanisms of rotational core losses.

(4) Preparation of the specimen is much simpler.

(5) This system can be conveniently incorporated into a system for domain structure observation, which is very important for understanding the mechanisms of rotational core losses.

The major drawback of this system is that it is difficult to control the flux density waveforms on the $X$ and $Y$ axes to be sinusoidal when the sample is close to saturation.

\section{Large Sheet Samples}

In 1991, Sievert and Enokizono developed a vertical yoke system as shown in Fig. 21, which allowed measurements on larger sheet samples $[40,54,56]$. Both the field-metric and watt-metric methods were used for rotational core loss evaluation, but the systematic error of the watt-metric method was high, up to $28 \%$ in the worst case [40]. The major problem, however, was the inability to reach high flux density values in the centre of the sample, where the rotational core loss was measured. It was stated that the highest flux density that could be reached in the sample was normally about $0.2 \mathrm{~T}$ lower than that achieved by the horizontal arrangement using square sample. According to Sievert [54], this was due to the heavy leakage and stray magnetic fluxes between the yokes of the $X$ and $Y$ axes.

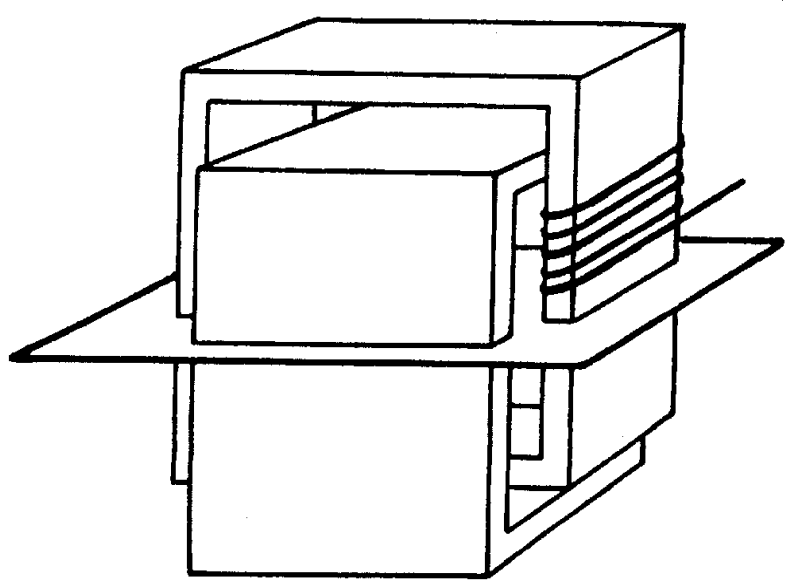

Fig. 21. Apparatus for 2-D measurements with vertical yokes [56]

\section{3-D MAgnetic Property Testing System}

In some electromagnetic devices such as a claw pole/transverse flux motor, the local flux density locus may follow an irregular loop in 3-D space when the rotor rotates $[55,57]$. In fact, even under 1-D alternating or 2-D rotating magnetic excitation, a magnetic material shows 3-D magnetic property due to the rotation of magnetic domains [58]. To investigate the 3-D magnetic properties of magnetic materials, a 3-D magnetic testing system was developed by the authors in 2001, as shown in Fig. 22 [27, 58-59]. By controlling the magnetic excitation in three axes, this tester is able to generate different magnetic flux patterns, such as alternating in any given orientation, rotating in a plane tilted for a given angle from an axis, and rotating in a 3-D pattern with the loci of the flux density vector tip forming a specified surface, according to the requirement of the test [30].

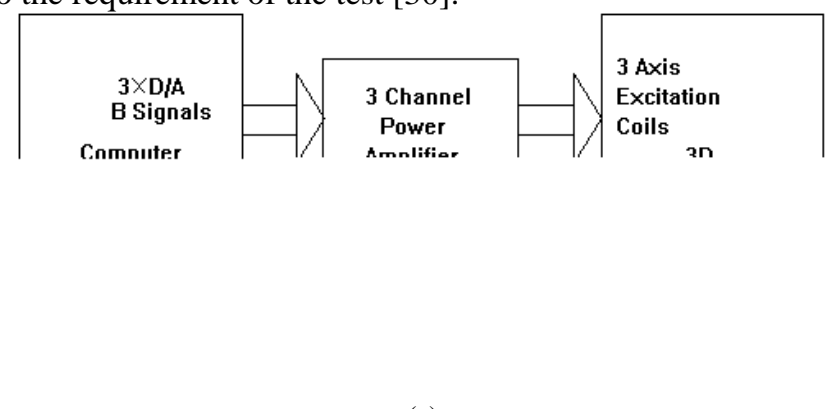

(a)

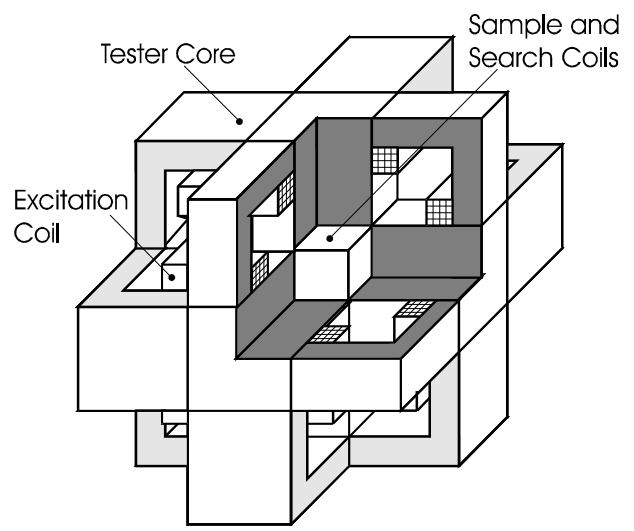

(b)

Fig. 22. 3-D magnetic tester: (a) testing system, and (b) frame [27]

\section{CONCLUSION AND DISCUSSION}

This paper aims to present an extensive review on the understanding, measurement and modeling of core losses of soft magnetic materials under rotating flux excitations in the past hundred years, based on a number of selected references. Although quite a few rotational core loss measurements and domain structure observations have been carried out on different ferromagnetic materials by various researchers, the mechanisms of rotational core loss are still far from being fully understood.

Due to the very complicated mechanisms, it is not practical to develop a model of strong physical background at the current stage. On the other hand, a phenomenological approach, such as the three term model generalized by Fiorillo from the corresponding alternating core loss model, appears to be useful for core loss analysis in rotating electrical machines.

Similar to the case of alternating core loss, the total rotational core loss can generally be separated into three components: rotational hysteresis, eddy current and anomalous losses. The ratio of rotational hysteresis loss to alternating hysteresis loss at low fields varies from one to two for different 
materials as reported by various researchers. With a circular field, the rotational eddy current loss is twice as much as alternating eddy current loss. The rotational anomalous loss can be modeled using the same formula as for alternating anomalous loss, but the coefficient of rotational anomalous loss is generally a function of flux density, and eventually reduces to zero when the material is saturated and all domain walls disappear.

Four major methods for measuring the rotational core loss are reviewed. The field-metric method is generally preferred as it features high accuracy and great versatility.

In order to further understand the mechanism of rotational core losses, considerable work for measurement experiment and theoretical modeling needs to be developed and to be improved, such as the following aspects: (1) Improvement of rotational core loss measuring techniques, including improved design of the tester with optimum magnetic circuit for more accurate measurement of the $\boldsymbol{B}-\boldsymbol{H}$ relationship; (2) New control strategy for controlling the locus of rotating flux density vector when the specimen is saturated; (3) Development of new modeling and measurement method of rotational core losses, including calculation of hysteresis and eddy current as well as anomalous core losses; and (4) Further understanding of the mechanisms of rotational hysteresis loss, including vector hysteresis modeling and experimental verification of the theory.

\section{REFERENCES}

[1] F. G. Baily, "The hysteresis of iron and steel in a rotating magnetic field," Phil. Trans. Royal Soc. A, vol. 187, pp. 715-746, 1896.

[2] F. Brailsford, "Rotational hysteresis loss in electrical sheet steels," Journal of IEE, vol. 83, pp. 566-575, 1938.

[3] W. F. Archenhold, H. F. Sandham, and J. E. Thompson, "Rotational hysteresis loss in grain-oriented silicon-iron," British Journal of Applied Physics, vol.11, pp. 46-49, Jan. 1960.

[4] C. R. Boon and J. E. Thompson, "Alternating and rotational power loss at $50 \mathrm{c} / \mathrm{s}$ in $3 \%$ silicon-iron sheets," Proc. of IEE, vol. 112, no. 11, pp. 2147-2151, Nov. 1965.

[5] F. Fiorillo and A. M. Reitto, "Extended induction range analysis of rotational losses in soft magnetic materials," IEEE Trans. Magn., vol. 24, no. 2, pp. 1960-1962, Mar. 1988.

[6] F. Fiorillo, "A phenomenological approach to rotational power losses in soft magnetic laminations," in Proc. ${ }^{\text {st }}$ Int. Workshop on Magnetic Properties of Electrical Sheet Steel under 2-D Excitation, PhysikalischTechnische Bundesanstalt (PTB), Braunschweig, Germany, 16-17 Sep. 1991, pp. 11-24.

[7] G. S. Radley, "Domain observation and wall velocity measurement under rotating field in grain-oriented silicon iron," in Proc. $1^{\text {st }}$ Int. Workshop on Magnetic Properties of Electrical Sheet Steel under 2-D Excitation, Physikalisch-Technische Bundesanstalt (PTB), Braunschweig, Germany, Sept. 1991, pp. 25-35.

[8] J. G. Zhu and V. S. Ramsden, "Improved formulations for rotational core losses in rotating electrical machines," IEEE Trans. Magn., vol. 34, no. 4, pp. 2234-2242, July 1998.

[9] A. Kaplan, "Magnetic core losses resulting from a rotating flux," Journal of Applied Physics, vol. 32, no. 3, pp. 370-371, Mar. 1961.

[10] R. D. Strattant and F. J. Young, "Iron losses in elliptical rotating fields," J. Applied Physics, vol. 33, no. 3, pp. 1285-1286, Mar. 1962.

[11] A. J. Moses and B. Thomas, "The spatial variation of localized power loss in two practical transformer T-joints," IEEE Trans. Magn., vol. 9, no. 4, pp. 655-659, Dec. 1973.

[12] R. Phillips and K. J. Overshott, "Domain configuration under rotational flux and applied stress conditions in silicon-iron," IEEE Trans. Magn., vol. 10, no. 2, pp. 168-169, June 1974.
[13] A. Basak and A. J. Moses, "Influence of stress on rotational loss in silicon iron," Proc. of IEE, vol. 125, no. 2, pp. 165-168, Feb. 1978.

[14] A. Cecchitti, G. Ferrari, F. Masoli, and G. P. Soardo, "Rotational power losses in 3\% SiFe as a function of frequency," IEEE Trans. Magn., vol. 14, no. 5, pp. 356-358, Sep. 1978.

[15] K. S. Tan, A. Datta, P. J. Flanders, and C. D. Graham, Jr., "Rotational loss in thin gauge soft magnetic materials," IEEE Trans. Magn., vol. 21, no. 5, pp. 1921-1923, Sep. 1985.

[16] P. J. Flanders, "A Hall sensing magnetometer for measuring magnetisation, anisotropy, rotational loss and time effects," IEEE Trans. Magn., vol. 21, no. 5, pp. 1584-1589, Sep. 1985.

[17] A. Kedous-Lebouc, S. Zouzou, and P. Brissonneau, "On the magnetisation processes in electrical steel in unidirectional and rotational field," in Proc. $1^{\text {st }}$ Int. Workshop on Magnetic Properties of Electrical Sheet Steel under 2-D Excitation, Physikalisch-Technische Bundesanstalt (PTB), Braunschweig, Germany, Sept. 1991, pp. 36-47.

[18] S. Zouzou, A. Kedous-Lebous, and P. Brissonneau, "Magnetic properties under unidirectional and rotational field," J. Magnetism and Magnetic Materials, vol. 112, pp. 106-108, 1992.

[19] A. J. Moses, "Rotational magnetization - problems in experimental and theoretical studies of electrical stress and amorphous magnetic materials," IEEE Trans. Magn., vol. 30, no. 2, pp. 902-906, Mar. 1994.

[20] K. Mori, S. Yanase, Y. Okazaki, and S. Hashi, "2-D magnetic rotational loss of electrical steel at high magnetic flux density," IEEE Trans. Magn., vol. 41, no. 10, pp. 3310-3312, Oct. 2005.

[21] M. Enokizono, T. Suzuki, and J. D. Sievert, "Measurement of dynamic magnetostriction under rotating magnetic field," IEEE Trans. Magn., vol. 26, no. 5, pp. 2067-2069, Sep. 1990.

[22] M. Enokizono, T. Suzuki, and J. Sievert, "Measurement of iron loss using rotational magnetic loss measurement apparatus," J. Applied Magn. in Japan (in Japanese), vol. 14, no. 2, pp. 455-458, 1990.

[23] M. Enokizono, G. Shirakawa, T. Suzuki, and J. Sievert, "Twodimensional magnetic properties of silicon steel sheet," J. Applied Magn. in Japan (in Japanese), vol. 15, no. 2, pp. 265-270, 1991.

[24] F. Fiorillo and A. M. Rietto, "The measurement of rotational losses at I.E.N.: Use of the thermometric method," in Proc. $1^{\text {st }}$ Int. Workshop on Magnetic Properties of Electrical Sheet Steel under 2-D Excitation, Physikalisch-Technische Bundesanstalt (PTB), Braunschweig, Germany, Sept. 1991, pp. 162-172.

[25] F. Fiorillo and A. M. Rietto, "Rotational and alternating energy loss vs. magnetising frequency in SiFe laminations," J. Magnetism and Magnetic Materials, vol. 83, pp. 402-404, 1990.

[26] J. G. Zhu, J. J. Zhong, V. S. Ramsden, and Y. G. Guo, "Power losses of composite soft magnetic materials under two dimensional excitations," J. Applied Physics, vol. 85, no. 8, pp. 4403-4405, Apr. 1999.

[27] J. J. Zhong, "Measurement and modelling of magnetic properties of materials with rotating fluxes," Ph.D. Thesis, University of Technology, Sydney, Australia, Dec. 2002.

[28] J. J. Zhong, Y. G. Guo, J. G. Zhu, and Z. W. Lin, "Characteristics of soft magnetic composite material under rotating fluxes," J. Magnetism and Magnetic Materials, vol. 299, no. 1, pp. 29-34, Apr. 2006.

[29] Y. G. Guo, J. G. Zhu, and J. J. Zhong, "Measurement and modelling of magnetic properties of soft magnetic composite materials under 2D vector magnetisations," J. Magnetism and Magnetic Materials, vol. 302, no. 1, pp. 14-19, July 2006.

[30] Y. G. Guo, J. G. Zhu, Z. W. Lin, and J. J. Zhong, "3D vector magnetic properties of soft magnetic composite material," J. Magnetism and Magnetic Materials, vol. 302, no. 2, pp. 511-516, July 2006.

[31] "The latest development in soft magnetic composite technology," $S M C$ Update, Reports of Höganäs AB, Sweden, 1997-2007. Available at http://www.hoganas.com/, see News then SMC Update.

[32] Y. G. Guo, J. G. Zhu, and W. Wu, "Thermal analysis of SMC motors using a hybrid model with distributed heat sources," IEEE Trans. Magn., vol. 41, no. 6, pp. 2124-2128, June 2005.

[33] E. Reisinger, "Measurement of iron losses due to alternating and rotating magnetization," in Proc. Electric Energy Conf., Adelaide, Australia, Oct. 6-9, 1987, pp. 388-392.

[34] A. J. Moses and B. Thomas, "Measurement of rotating flux in silicon iron laminations," IEEE Trans. Magn., vol. 9, No. 4, pp. 651-654, Dec. 1973.

[35] W. Brix, K. A. Hempel, and W. Schroeder, "Method for the measurement of rotational power loss and related properties in electrical 
steel sheets," IEEE Trans. Magn., vol. 18, no. 6, pp. 1469-1471, Nov. 1982.

[36] J. Sievert, "On measuring the magnetic properties of electrical sheet steel under rotational magnetization," J. Magnetism and Magnetic Materials, vol. 112, pp. 50-57, 1992.

[37] J. G. Zhu, "Numerical modeling of magnetic materials for computer aided design of electromagnetic devices," Ph.D. Thesis, University of Technology, Sydney, Australia, July 1994.

[38] Y. Alinejad-Beromi, A. J. Moses, and T. Meydan, "New aspects of rotational field and flux measurement in electrical steel," J. Magnetism and Magnetic Materials, vol. 112, pp. 135-138, 1992.

[39] International Electrotechnical Commission, "Magnetic materials, Part 3: Methods of measurements of the magnetic properties of magnetic sheet and strip by means of a single sheet tester," 404-3 IEC.

[40] J. Sievert, H. Ahlers, M. Enokizono, S. Kauke, L. Rahf, and J. Xu, "The measurement of rotational power loss in electrical sheet steel using a vertical yoke system," J. Magnetism and Magnetic Materials, vol. 112, pp. 91-94, 1992.

[41] M. Enokizono and J. Sievert, "Numerical analysis of accuracy of rotational magnetic loss measurement apparatus," IEEE Translation J. Magn. in Japan, vol. 5, no. 9, pp. 742-748, Sept. 1990.

[42] J. Sievert, "Recent advances in the one- and two-dimensional magnetic measurement technique for electrical sheet steel," IEEE Trans. Magn., vol. 26, no. 5, pp. 2553-2558, Sept. 1990.

[43] J. G. Zhu and V. S. Ramsden, "Two dimensional measurement of magnetic field and core loss using square specimen tester," IEEE Trans. Magn., vol. 29, no. 6, pp. 2995-2997, Nov. 1993.

[44] F. J. Schulte, "Eine meßeinrichtung zur zweidimensionalen erfassung der magnetischen eignschaften von elektroblech," Dissertation RWTH Aachen, 1989, Fakultät für Elektrotechnik.

[45] W. Salz and K. A. Hempel, "Which field sensors are suitable for a rotating flux apparatus," in Proc. $1^{\text {st }}$ Int. Workshop on Magnetic Properties of Electrical Sheet Steel under 2-D Excitation, PhysikalischTechnische Bundesanstalt (PTB), Braunschweig, Germany, Sept. 1991, pp. 117-126.

[46] J. J. Zhong, J. G. Zhu, V. S. Ramsden, and Y. G. Guo, "Magnetic properties of composite soft magnetic materials with 2-D fluxes," in Proc. Australasian Universities Power Engineering Conf., Hobart, Australia, Sept. 27-30, 1998, pp. 377-382.

[47] E. Werner, Austria patent No. 19115, 1949.

[48] J. Sievert, J. Xu, L. Rahf, M. Enokizono, and H. Ahlers, "Studies on the rotational power loss measurement problem," Anales de Fisica Serie B, vol. 86, pp. 35-37, 1990.

[49] P. J. Flanders, "The rotating-sample magnetometer," J. Applied Physics, vol. 38, no. 3, pp. 1293-1294, Mar. 1967.

[50] F. Fiorillo and A. M. Rietto, "The measurement of rotational losses at I.E.N.: Use of the thermometric method," in Proc. $1^{\text {st }}$ Int. Workshop on Magnetic Properties of Electrical Sheet Steel under 2-D Excitation, Physikalisch-Technische Bundesanstalt (PTB), Braunschweig, Germany, Sept. 1991, pp. 162-172.

[51] T. Sasaki, M. Imamura, S. Takada, and Y. Suzuki, "Measurement of rotational power losses in silicon-iron sheets using wattmeter method," IEEE Trans. Magn., vol. 21, no. 5, pp. 1918-1920, Sept. 1985.

[52] W. Brix, K. A. Hempel, and F. J. Schulte, "Improved method for the investigation of the rotational magnetization process in electrical steel sheets," IEEE Trans. Magn., vol. 20, no. 5, pp. 1708-1710, Sept. 1984.

[53] M. Enokizono, "Studies on two-dimensional magnetic measurement and properties of electrical steel sheets at Oita University," in Proc. $1^{\text {st }}$ Int. Workshop on Magnetic Properties of Electrical Sheet Steel under 2-D Excitation, Physikalisch-Technische Bundesanstalt (PTB), Braunschweig, Germany, Sept. 1991, pp. 82-101.

[54] J. Sievert, "Studies on the measurement of two dimensional magnetic phenomena in electrical sheet steel at PTB," in Proc. $1^{\text {st }}$ Int. Workshop on Magnetic Properties of Electrical Sheet Steel under 2-D Excitation, Physikalisch-Technische Bundesanstalt (PTB), Braunschweig, Germany, Sept. 1991, pp. 102-116.

[55] Y. G. Guo, "Development of low cost high performance permanent magnet motors using new soft magnetic materials," Ph.D. Thesis, University of Technology, Sydney, Australia, Dec. 2003.

[56] M. Enokizono, T. Todaka, T. Sashikata, J. D. Sievert, and H. Ahlers, "Magnetic field analysis of rotational loss tester with vertical yoke," $J$. Magnetism and Magnetic Materials, vol. 112, pp. 81-84, 1992.
[57] Y. G. Guo, J. G. Zhu, Z. W. Lin, and J. J. Zhong, "Measurement and modeling of core losses of soft magnetic composites under 3D magnetic excitations in rotating motors," IEEE Trans. Magn., vol. 41, no. 10, pp. 3925-3927, Oct. 2005.

[58] J. G. Zhu, J. J. Zhong, Z. W. Lin, and J. D. Sievert, "Measurement of magnetic properties under 3-D magnetic excitations," IEEE Trans. Magn., vol. 39, no. 5, pp. 3429-3431, Sept. 2003.

[59] J. J. Zhong and J. G. Zhu, "Electromagnetic design of a 3D tester for magnetic properties of soft magnetic materials," in Proc. $5^{\text {th }}$ Int. Conf. on Electrical Machines and Systems, Shenyang, China, Sept. 2001, pp. 392-395.

Youguang Guo (S'02-M'05-SM'06) was born in Hubei, China in 1965. He received the B.E. degree from Huazhong University of Science and Technology (HUST), China in 1985, the M.E. degree from Zhejiang University, China in 1988, and the $\mathrm{PhD}$ degree from University of Technology, Sydney (UTS), Australia in 2004, all in electrical engineering.

From 1988 to 1998, he lectured in the Department of Electric Power Engineering, HUST. From March 1998 to July 2000, he was a visiting research fellow in the Center for Electrical Machines and Power Electronics, Faculty of Engineering, UTS. He is currently an ARC (Australia Research Council) postdoctoral research fellow at UTS.

His research fields include measurement and modeling of magnetic properties of magnetic materials, numerical analysis of electromagnetic field, motor design and optimization, power electronics and control of electrical appliance. He has published over 160 refereed technical papers including 81 journal articles.

Jian Guo Zhu (S'93-M'96-SM'03) is the Professor of Electrical Engineering and Director for the Center for Electrical Machines and Power Electronics at University of Technology, Sydney (UTS), Australia. He received his BE in 1982 from Jiangsu Institute of Technology, China, ME in 1987 from Shanghai University of Technology, China, and Ph.D in 1995 from UTS, Australia. His research interests are: electromagnetics, magnetic properties of materials, electrical machines and drives, power electronics, and renewable energy systems.

Jinjiang Zhong was born in Heilongjiang Province, China, in 1963. He received the B.E. from Wuhan University of Science and Technology, China in 1987, and Ph.D. from University of Technology, Sydney (UTS), Australia in 2003. He is currently a research fellow in the Center for Electrical Machines and Power Electronics, UTS. His research interest includes measurement and modeling of magnetic properties of magnetic materials under 2-D and 3-D vector magnetizations.

Haiyan Lu is with the Faculty of Information Technology, University of Technology, Sydney (UTS). She gained her Bachelor and Master of Electrical Engineering in Harbin Institute of Technology, China in 1985 and 1988, respectively. She achieved her $\mathrm{PhD}$ in Faculty of Engineering, UTS, Australia, in 2002. Her current research interests are optimal design of electromagnetic devices, modeling and numerical 
simulation of magnetic materials, soft computing techniques and applications in power systems and power market.

Jian Xun Jin was born in Beijing, in 1962. He received B.Eng. degree from Beijing University of Science and Technology in 1985, M.Sc. degree from University of New South Wales, Australia in 1994, and Ph.D. degree from University of Wollongong, Australia in 1997. He was a research fellow and Australian ARC project chief investigator and senior research fellow with Australian University of
Wollongong from 1997 to 2003 . He has published over 300 papers as an author and co-author mainly on applied high temperature superconductivity. He is currently a professor and the Director of the Center of Applied Superconductivity and Electrical Engineering, University of Electronic Science and Technology of China. His research interests include applied high temperature superconductivity and energy efficiency technology. 